\title{
A COMPARISON TECHNIQUE FOR INTEGRAL EQUATIONS ${ }^{1}$
}

\author{
Maria Meehan \& Donal O'Regan
}

\section{Introduction}

In this note we survey some results for integral equations on the infinite interval. We are interested in existence results for nonlinear Volterra integral equations, and in particular, results that enable us to study aspects of the solution's behaviour on the interval of existence.

In Section 2 we examine the nonlinear Volterra integral equation

$$
y(t)=h(t)-\int_{0}^{t} k(t, s) g(s, y(s)) d s, \quad t \in[0, T)
$$

where $0 \leq T \leq \infty$. Existence results are given which establish a solution $y \in C[0, T)$ of (1.1) and yield functions $a, b \in C[0, T)$ such that $a(t) \leq y(t) \leq b(t)$, for $t \in[0, T)$. Obviously the behaviour of $a$ and $b$ on $[0, T)$ will in many cases allow us to extract additional information on the solution $y$. It is this fact that we exploit in Section 3.

Using the results obtained for (1.1) in Section 2, a comparison technique is presented in Section 3 which firstly guarantees that the solution $y \in C[0, T)$ of (1.1) (with $T=\infty$ ), is such that $\lim _{t \rightarrow \infty} y(t)$ exists, and secondly, allows us to read off what this limit is. The technique is illustrated with some examples. A result of Miller [9], which pertains to a special case of (1.1) is included and discussed for completeness.

${ }^{1}$ The results of this paper were presented by the first author at the Science Research Colloquium, I.T. Tallaght, 25-27 May, 1998. 
We conclude this section by stating some definitions which will be used throughout the paper. Let $I$ be an interval in $\mathbf{R}$.

Definition 1.1: A function $g: I \times \mathbf{R} \rightarrow \mathbf{R}$ is a $L^{q}$-Carathéodory function if the following conditions hold:

(i) the map $t \mapsto g(t, y)$ is measurable for all $y \in \mathbf{R}$;

(ii) the map $y \mapsto g(t, y)$ is continuous for almost all $t \in I$;

(iii) for any $r>0$, there exists $\mu_{r} \in L^{q}(I)$ such that $|y| \leq r$ implies that $|g(t, y)| \leq \mu_{r}(t)$ for almost all $t \in I$.

Definition 1.2: A function $g: I \times \mathbf{R} \rightarrow \mathbf{R}$ is a locally $L^{q}$ Carathéodory function if the conditions in Definition 1.1 hold when $g$ is restricted to $J \times \mathbf{R}$, with $J$ any compact subinterval of $I$.

\section{Existence results}

Before presenting our existence results for

$$
y(t)=h(t)-\int_{0}^{t} k(t, s) g(s, y(s)) d s, \quad t \in[0, T)
$$

where $0 \leq T \leq \infty$, we first state the following variation of an existence principle of Lee and O'Regan [7], which we will require in this section. For details of the proof we refer the reader to $[7,8]$.

Theorem 2.1. Let $0 \leq T \leq \infty$, and suppose that $p$ and $q$ satisfy $1 \leq p \leq \infty$ and $\frac{1}{p}+\frac{1}{q}=1$. Assume that

$$
h \in C[0, T)
$$

$$
g:[0, T) \times \mathbf{R} \rightarrow \mathbf{R} \text { is locally } L^{q}-\text { Carathéodory }
$$

[see Definition 1.2]

$$
k_{t}(s)=k(t, s) \in L^{p}[0, t] \text { for each } t \in[0, T)
$$

and for any $t, t^{\prime} \in[0, T)$,

$$
\int_{0}^{t^{\star}}\left|k_{t}(s)-k_{t^{\prime}}(s)\right|^{p} d s \rightarrow 0 \text { as } t \rightarrow t^{\prime}, \text { where } t^{\star}=\min \left\{t, t^{\prime}\right\}
$$


hold. Also let $0<t_{1}<t_{2}<\ldots<t_{n}<\ldots$, with $t_{n} \uparrow T$. Suppose for each $n=1,2, \ldots$, that there exists $y_{n} \in C\left[0, t_{n}\right]$ that solves

$$
y_{n}(t)=h(t)-\int_{0}^{t} k(t, s) g\left(s, y_{n}(s)\right) d s, t \in\left[0, t_{n}\right],
$$

and that there are bounded sets $B_{k} \subseteq \mathbf{R}$, for $k=1,2, \ldots$, such that $n \geq k$ implies $y_{n}(t) \in B_{k}$ for each $t \in\left[0, t_{k}\right]$. Then (2.1) has a solution $y \in C[0, T)$ such that $y(t) \in B_{k}$ for each $t \in\left[0, t_{k}\right]$.

NOTE: For $p=\infty$, let

$$
\left(\int_{0}^{T}|f(s)|^{p} d s\right)^{\frac{1}{p}} \text { denote ess-sup } s_{s \in[0, T]}|f(s)| .
$$

Our first two existence results for (2.1), which extend the results of Friedman [3] and Miller [9] in the literature, give conditions under which (2.1) has a solution $y \in C[0, T)$ and is bounded by two functions $a, b \in C[0, T)$. Of particular importance is the information which we can obtain on the positivity of the solution.

Theorem 2.2. Let $1 \leq p \leq \infty$ be a constant, and $q$ be such that $\frac{1}{p}+\frac{1}{q}=1$. Assume that $(2.2)-(2.5)$ hold. Suppose also that

$$
k(t, s) \geq 0 \text { for almost every } 0 \leq s \leq t<T
$$

$$
\left\{\begin{array}{l}
\text { for } 0 \leq t_{0} \leq t_{1}<T \text { and } s \in\left[0, t_{0}\right] \\
k\left(t_{0}, s\right) \frac{h\left(t_{1}\right)}{h\left(t_{0}\right)} \geq k\left(t_{1}, s\right), \text { if } h\left(t_{0}\right) \neq 0 \\
k\left(t_{0}, s\right) \geq k\left(t_{1}, s\right), \text { if } h(t)=0, t \in\left[t_{0}, t_{1}\right]
\end{array}\right.
$$

$$
\left\{\begin{array}{l}
\text { there exists a nonincreasing function } r \in C[0, T) \\
\text { such that for almost every } s \in[0, T), g(s, r(s))=0
\end{array}\right.
$$


and one of the following holds:

$$
h \text { is nondecreasing and } h(t) \geq r(t) \geq 0, t \in[0, T)
$$

$$
h \text { is nondecreasing and } 0 \geq h(t) \geq r(t), t \in[0, T)
$$

$$
h \text { is nonincreasing and } h(t) \geq 0 \geq r(t), \quad t \in[0, T)
$$

along with

$$
\left\{\begin{array}{c}
g(s, u) \geq 0 \text { for } h(s) \geq u \geq r(s), \\
\text { and almost every } s \in[0, T)
\end{array}\right.
$$

and

$$
\left\{\begin{array}{l}
r(0) \leq \lim _{t \rightarrow 0^{+}}\left(h(t)-\int_{0}^{t} k(t, s) g(s, u(s)) d s\right), \\
\text { for all } u \in C[0, T) \text { such that } \\
h(s) \geq u(s) \geq r(s), s \in[0, T) .
\end{array}\right.
$$

Then there exists a solution $y \in C[0, T)$ of $(2.1)$ with $h(t) \geq y(t) \geq$ $r(t), t \in C[0, T)$.

Proof: For details of the proof (which relies on Theorem 2.1 and Schauder's Fixed Point Theorem) we refer the reader to [8].

Remark 2.1. Suppose that the conditions in Theorem 2.2 hold with $r \equiv 0$. Then notice from (2.9) and (2.11), that whether $h$ is nondecreasing or nonincreasing, as long as it is nonnegative, we have a solution of (2.1). In fact, closer examination reveals that we can find a solution of (2.1), even if $h$ is not monotonic. We state the result as the following corollary. See [8] for details of the proof.

Corollary 2.1. Let $1 \leq p \leq \infty$ be a constant, and let $q$ be such that $\frac{1}{p}+\frac{1}{q}=1$. Assume that $(2.2)-(2.5)$ hold and suppose that (2.6),

$$
h>0 \text { on }[0, T)
$$




$$
\left\{\begin{array}{l}
\text { for } 0 \leq t_{0} \leq t_{1}<T \text { and } s \in\left[0, t_{0}\right] \\
k\left(t_{0}, s\right) \frac{h\left(t_{1}\right)}{h\left(t_{0}\right)} \geq k\left(t_{1}, s\right)
\end{array}\right.
$$

$$
\left\{\begin{array}{l}
g \text { is such that } y g(s, y) \geq 0, \text { for all } y \in \mathbf{R} \\
\text { and almost every } s \in[0, T)
\end{array}\right.
$$

and

$$
\left\{\begin{array}{l}
0 \leq \lim _{t \rightarrow 0^{+}}\left(h(t)-\int_{0}^{t} k(t, s) g(s, u(s)) d s\right) \\
\text { for all } u \in C[0, T) \text { such that } h(s) \geq u(s) \geq 0
\end{array}\right.
$$

hold. Then there exists a solution $y \in C[0, T)$ of $(2.1)$ with $h(t) \geq$ $y(t) \geq 0$, for $t \in[0, T)$.

Notice that in the hypotheses of Theorem 2.2 we have $h(t) \geq r(t)$, for $t \in[0, T)$. If the opposite was true, then we have the following theorem which we just state. The ideas in the proof are similar to those in the proof of Theorem 2.2.

Theorem 2.3. Let $1 \leq p \leq \infty$ be a constant, and let $q$ be such that $\frac{1}{p}+\frac{1}{q}=1$. Assume that (2.2) $-(2.5)$ hold, and that (2.6) and (2.7) are true. In addition suppose that

$$
\left\{\begin{array}{l}
\text { there exists a nondecreasing function } r \in C[0, T) \\
\text { such that for almost every } s \in[0, T), g(s, r(s))=0
\end{array}\right.
$$

and one of the following holds:

$$
h \text { is nonincreasing and } r(t) \geq h(t) \geq 0, t \in[0, T)
$$$$
h \text { is nonincreasing and } 0 \geq r(t) \geq h(t), t \in[0, T)
$$

$$
h \text { is nondecreasing and } r(t) \geq 0 \geq h(t), t \in[0, T)
$$


along with

$$
\left\{\begin{array}{c}
g(s, u) \leq 0 \text { for } r(s) \geq u \geq h(s), \\
\text { and almost every } s \in[0, T)
\end{array}\right.
$$

and

$$
\left\{\begin{array}{l}
r(0) \geq \lim _{t \rightarrow 0^{+}}\left(h(t)-\int_{0}^{t} k(t, s) g(s, y(s)) d s\right) \\
\text { for all } u \in C[0, T) \text { such that } r(s) \geq u(s) \geq h(s) .
\end{array}\right.
$$

Then there exists a solution $y \in C[0, T)$ of $(2.1)$ with $r(t) \geq y(t) \geq$ $h(t), t \in[0, T)$.

NOTE: If $r=0$ in Theorem 2.3, a remark analogous to Remark 2.1 can be made for Theorem 2.3.

Remark 2.2. If $h(t) \equiv h_{0}$ and $r(t) \equiv r_{0}$, where $h_{0}$ and $r_{0}$ are constants, Theorem 2.2 and Theorem 2.3 instantly yield some simple results.

The first, perhaps obvious one, is that if $h_{0}=r_{0}$, then immediately we see that $y=h_{0}=r_{0}$ is a solution of (2.1). Secondly, suppose $g$ is such that

$$
\left(y-r_{0}\right) g\left(s, y-r_{0}\right) \geq 0, \text { for almost every } s \in[0, T) .
$$

Then for any $h_{0},(2.1)$ has a solution $y \in C[0, T)$. For example,

$$
y(t)=h_{0}-\int_{0}^{t} \frac{r_{0}^{n}-y^{n}(s)}{(t-s)^{\alpha}} d s, t \in[0, T)
$$

where $h_{0}$ is any constant, $0<\alpha<1$, and $n \geq 0$ is odd.

In fact, if $h, k$ and $g$ satisfy the hypotheses of Theorem 2.2 and $g$ is odd, that is $g(s,-u)=-g(s, u)$ for almost every $s \in[0, T)$, then $h_{1}, k$ and $g_{1}$, where $h_{1}=-h$, and $g_{1}=-g$, satisfy the hypotheses of Theorem 2.3.

Remark 2.3. Since in both Theorem 2.2 and Theorem 2.3, we have that our solution $y \in C[0, T)$ is bounded by two functions 
$h, r \in C[0, T)$, we can often extract additional information about $y$ when given more details about $h$ and $r$. For example, if $h, r \in$ $B C[0, T)$, then obviously $y \in B C[0, T)$. A more interesting result, and indeed one which leads us on naturally to the final section is the following:

Suppose the hypotheses of Theorem 2.2 hold, with $r=0, h$ nonincreasing and $h \in C_{0}[0, T)$. [Here $h \in C_{0}[0, T)$ if $h \in C[0, T)$ and $\lim _{t \rightarrow T} h(t)=0$.] Then we have a solution $y \in C[0, T)$ with $0 \leq y(t) \leq h(t)$. But notice by the Sandwich Theorem that $\lim _{t \rightarrow T} y(t)=0$ also, that is, $y \in C_{0}[0, T)$. [An analogous remark can be made for Theorem 2.3.] We give the following example:

Example 2.1. Let $n>0$ and consider

$$
y(t)=\frac{1}{\sqrt{t+1}}-\int_{0}^{t} \frac{1}{\sqrt{t-s}} y^{n}(s) d s, t \in[0, \infty) .
$$

Now (2.24) satisfies the hypotheses of Theorem 2.2 with (2.11) true. Therefore $(2.24)$ has a solution $y \in C[0, \infty)$ with $0 \leq y(t) \leq$ $\frac{1}{\sqrt{t+1}}, t \in[0, \infty)$, and clearly $y \in C_{0}[0, \infty)$.

\section{A comparison technique for integral equations}

We've already seen in the previous section that if $h, k$ and $g$ satisfy the hypotheses of Theorem 2.2 or Theorem 2.3, then

$$
y(t)=h(t)-\int_{0}^{t} k(t, s) g(s, y(s)) d s, \quad t \in[0, \infty)
$$

has a solution $y \in C[0, \infty)$, such that for $t \in[0, \infty), h(t) \geq y(t) \geq$ $r(t)$ or $r(t) \geq y(t) \geq h(t)$, respectively.

The questions which we now ask are:

Under what additional assumptions on $h, k$ and $g$, will (3.1) have a solution $y$ such that $\lim _{t \rightarrow \infty} y(t)$ exists? And can we find this limit?

We have already given one answer to each of these questions in Remark 2.3. For completeness, we state the result here. 
Corollary 3.1. Suppose the following are true:

$$
\left\{\begin{array}{c}
h, k \text { and } g \text { satisfy the hypotheses of either } \\
\text { Theorem } 2.2 \text { or Theorem } 2.3 \\
h, r \in C_{l}[0, \infty),
\end{array}\right.
$$

where $r$ is as defined in (2.8) and (2.18) respectively.

Then if $h(\infty)=r(\infty)$, (3.1) has a solution $y \in C_{l}[0, \infty)$ and $\lim _{t \rightarrow \infty} y(t)=h(\infty)=r(\infty)$.

Another approach is to use a comparison technique which we describe in the following theorem.

Theorem 3.1. Suppose that

(3.4) $\quad h, k$ and $g$ satisfy the hypotheses of Theorem 2.2

with the additional assumptions that

(3.5) $g(s, u)$ is nondecreasing in $u$ for almost every $s \in[0, \infty)$

(3.6) $\quad r \in C_{l}[0, \infty)$, where $r$ is as defined in (2.8)

$$
\left\{\begin{array}{l}
k \text { satisfies the following property: } \\
\text { For any nonnegative } \theta, \phi \in L_{l o c}^{q}[0, \infty), \text { the equation } \\
z(t)=\int_{0}^{t} k(t, s) \theta(s)-k(t, s) \phi(s) z(s) d s, t \in[0, \infty) \\
\text { has a unique, nonnegative solution } z \in C[0, \infty)
\end{array}\right.
$$

hold. Suppose also that

$$
\left\{\begin{array}{l}
\text { there exists a function } \\
\tilde{g}:[0, \infty) \times \mathbf{R} \rightarrow \mathbf{R}, \text { and } \tilde{r} \in C[0, \infty) \\
\text { such that } \tilde{g}(s, \tilde{r}(s))=0 \text { and } \tilde{g}(s, u(s)) \leq g(s, u(s)) \\
\text { for almost every } s \in[0, \infty) \text { and for all } u \in C[0, \infty), \\
\text { that satisfy } h(s) \geq u(s) \geq \tilde{r}(s), s \in[0, \infty) . \\
\text { Suppose also that } \\
y(t)=h(t)-\int_{0}^{t} k(t, s) \tilde{g}(s, y(s)) d s, t \in[0, \infty) \\
\text { has a unique solution } \tilde{y} \in C[0, \infty), \text { such that } \\
h(t) \geq \tilde{y}(t) \geq \tilde{r}(t), \text { for } t \in[0, \infty) \text { and } \tilde{y}(\infty)=r(\infty)
\end{array}\right.
$$


is true. Then (3.1) has a unique solution $y \in C_{l}[0, \infty)$, with $h(t) \geq$ $y(t) \geq r(t), t \in[0, \infty)$ and $y(\infty)=r(\infty)$.

Proof: For details of the proof we refer the reader to [8].

The following result which is analogous to Theorem 3.1, we state without proof.

Theorem 3.2. Suppose that

$h, k$ and $g$ satisfy the hypotheses of Theorem 2.3

and $\quad r \in C_{l}[0, \infty)$, where $r$ is as defined in (2.18)

hold, along with (3.5) and (3.7). Suppose also that

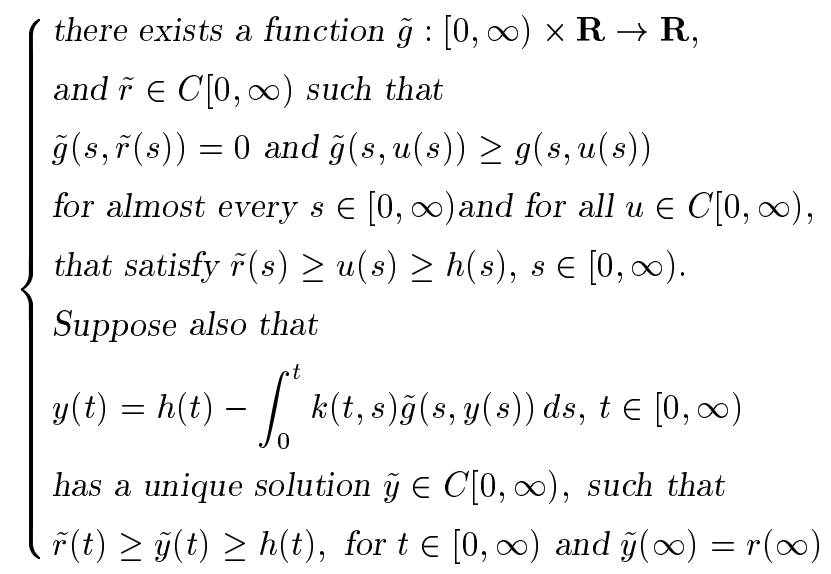

is true. Then (3.1) has a unique solution $y \in C_{l}[0, \infty)$ with $r(t) \geq$ $y(t) \geq h(t)$, for $t \in[0, \infty)$ and $y(\infty)=r(\infty)$.

Some of the hypotheses in the last two theorems warrant further discussion. In particular, (3.7), (3.8) and (3.11) need to be examined. We first look at (3.7).

In [9], Miller proves that if the kernel $k$ is of convolution type, that is, $k(t, s)=a(t-s)$, and the function $a(t)$ satisfies

$$
a \in L^{1}(0,1)
$$


(3.13) $\quad a$ is positive, continuous and nondecreasing on $(0, \infty)$

and

$$
\left\{\begin{array}{l}
\text { for any } T>0, \text { the function } \frac{a(t)}{a(t+T)} \\
\text { is a nonincreasing function of } t \text { on }(0, \infty),
\end{array}\right.
$$

then $k(t, s)=a(t-s)$ satisfies (3.7). The proof makes use of the resolvent kernel and its associated properties when $a$ satisfies $(3.12)-(3.14)$.

Remark 3.1. It is worth noticing that (3.14) holds if and only if $\log (a(t))$ is convex.

An example of such a function is $a(t)=t^{-\alpha}$, where $0 \leq$ $\alpha<1$. The singular convolution kernel associated with this $a$, which also arises in Abel's equation, is of interest to us. In particular, when $\alpha=\frac{1}{2}$, we have the kernel which arises in the well known tautochrone problem, and many satisfactory results can be obtained.

However, we wish to consider (3.7) for a more general kernel, $k$. Initially suppose that $k$ satisfies the hypotheses of either Theorem 2.2 or Theorem 2.3. Letting

$$
h^{\star}(t)=\int_{0}^{t} k(t, s) \theta(s) d s \text { and } k^{\star}(t, s)=k(t, s) \phi(s),
$$

we can rewrite

(3.15) $z(t)=\int_{0}^{t} k(t, s) \theta(s) d s-\int_{0}^{t} k(t, s) \phi(s) z(s) d s, t \in[0, \infty)$

as

$$
z(t)=h^{\star}(t)-\int_{0}^{t} k^{\star}(t, s) z(s) d s, t \in[0, \infty) .
$$


Note that $h^{\star} \in C[0, \infty)$ and for convenience, suppose $h^{\star}(t)>0$, for $t \in[0, \infty)$. If, for $0 \leq t_{0} \leq t_{1}<\infty, s \in\left[0, t_{0}\right]$, we have

$$
k^{\star}\left(t_{0}, s\right) \frac{h^{\star}\left(t_{1}\right)}{h^{\star}\left(t_{0}\right)} \geq k^{\star}\left(t_{1}, s\right),
$$

then by Corollary 2.1, (3.16) has a nonnegative solution $z \in$ $C[0, \infty)$. Clearly a separable kernel $k(t, s)=a(t) b(s)$, where $a(t)>0, b(t)>0$ for all $t \in[0, \infty)$, appropriately defines $h^{\star}$ and $k^{\star}$ (that is, (3.17) is true in this setting). Also for a separable kernel one can easily verify that the solution $z$ is unique.

In general, if,

$$
\left\{\begin{array}{l}
\text { in addition to the hypotheses of either Theorem 2.2 } \\
\text { Corollary } 2.1 \text { or Theorem } 2.3, k \text { satisfies } \\
k(v, s) k(t, u) \leq k(t, s) k(v, u), \text { for } s \leq u \leq v \leq t
\end{array}\right.
$$

holds, then (3.15) has a unique, nonnegative solution $z \in C[0, \infty)$. To see this, note by definition that $k^{\star}$ satisfies (3.18) also. Then Gripenberg, Londen and Staffans [4, Ch9, p.259], show that $k^{\star}$ has a nonnegative resolvent $r^{\star}$ where

$$
\begin{aligned}
r^{\star}(t, s) & =k^{\star}(t, s)-\int_{s}^{t} k^{\star}(t, u) r^{\star}(u, s) d u \\
& =k^{\star}(t, s)-\int_{s}^{t} r^{\star}(t, u) k^{\star}(u, s) d u,
\end{aligned}
$$

for almost every $(t, s) \in[0, \infty) \times[0, t]$, and $r^{\star}$ is strictly positive almost everywhere on the set where $k^{\star}$ is strictly positive. In addition, (3.16) has a unique solution given by

$$
z(t)=h^{\star}(t)-\int_{0}^{t} r^{\star}(t, s) h^{\star}(s) d s .
$$

Now an argument similar to Miller [9, p.329], shows that $z \geq 0$. 
We now turn our attention to (3.8). [Analogous comments can be made for (3.11).] Condition (3.8) requires us to have previous knowledge of the existence, uniqueness and asymptotic behavior of a solution $\tilde{y}$ of

$$
y(t)=h(t)-\int_{0}^{t} k(t, s) \tilde{g}(s, y(s)) d s, t \in[0, \infty) .
$$

At the outset, this may seem like an unreasonable condition since (3.19) closely resembles (3.1), the equation under examination. However, information on the solution $\tilde{y}$ to (3.19) is easily obtained if an appropriate choice of $\tilde{g}$ is made. For examples of "appropriate" $\tilde{g}$, we first look to the literature.

As already mentioned, Miller in [9] discusses (3.1) when the kernel $k$ is of convolution type, that is, $k(t, s)=a(t-s)$ and $a$ satisfies (3.12) - (3.14). He puts conditions on $a$ and $g$ so that

$$
y(t)=-\int_{0}^{t} a(t-s) g(s, y(s)) d s, t \in[0, \infty)
$$

has a unique, nonnegative, nondecreasing solution $y \in C[0, \infty)$ and then discusses when $y(\infty)=r(\infty)<\infty$. [Here again, $r$ is such that $g(s, r(s))=0$, for almost every $s \in[0, \infty)$.]

For completeness, we state his result.

Theorem 3.3. Suppose a satisfies (3.12) - (3.14), and $g$ satisfies

$$
\left\{\begin{array}{l}
g \text { is measurable in }(t, u) \\
\text { for } 0 \leq t<\infty,-\infty<u<\infty, \\
\text { continuous and nondecreasing in } u \text { for each fixed } t, \\
\text { nonincreasing in } t \text { for each fixed } u, \\
\text { and bounded on each finite rectangle } \\
y_{1} \leq u \leq y_{2}, 0 \leq t \leq t_{1} .
\end{array}\right.
$$

$$
\left\{\begin{array}{l}
\text { there is a function } r(t), \text { bounded on each } \\
\text { finite subinterval of }[0, \infty) \text { with } r(0) \geq 0 \\
\text { such that } g(t, r(t))=0 \text { for all } t \geq 0
\end{array}\right.
$$


and $g(t, y)$ is locally Lipschitz continuous in $y$.

Then the solution $y(t)$ of (3.20) is nonnegative and nondecreasing on $0 \leq t<\infty$.

In addition we have the following results:

Suppose that for each $\delta>0$, there exists $T>0$ and $\phi(\delta)>0$, such that $g(t, y) \leq \phi(\delta)$ if $t \geq T$ and $0<y<r(t)-\delta$. If $a \neq L^{1}(0, \infty)$ and $r(\infty)<\infty$, then the solution $y(t)$ of (3.20) tends to the limit $r(\infty)$ as $t \rightarrow \infty$.

or

Suppose that $g(t, y+r(t)) \rightarrow 0$ as $(t, y) \rightarrow\left(\infty, 0^{-}\right)$. If $a \in L^{1}(0, \infty)$ and $r(\infty)<\infty$, then the solution $y(t)$ of (3.20) tends to a limit $y(\infty)<r(\infty)$ as $t \rightarrow \infty$.

Remark 3.2. Note that by itself, Theorem 3.3 gives another answer to the two questions we asked at the beginning of this section.

Even though we can apply Theorem 3.3 in certain cases, we want to emphasize that Theorem 3.1 and Theorem 3.2 exist independently of results in the literature. We therefore initially consider examples when $\tilde{g}$ is a linear function of $y$, and it is easy to explicitly find the solution $\tilde{y}$ and in turn $\tilde{y}(\infty)$ from first principles. For example, we can use Laplace Transforms if we have a convolution kernel, or use the resolvent kernel if it is easy to find. Using these examples and Theorem 3.1 and Theorem 3.2, we can quickly build up a store of results from which we can extract information for (3.8) and (3.11). In addition, it is easy to construct examples where our theory applies, but Theorem 3.3 does not.

Example 3.1. Suppose that $a \in C^{1}[0, \infty), b \in C[0, \infty)$ and $a(t)>0, b(t)>0$ for all $t \in[0, \infty)$ and consider

$$
y(t)=-a(t) \int_{0}^{t} b(s)(y(s)-1) d s, t \in[0, \infty) .
$$


By Theorem 2.3, (3.24) has a solution $y \in C[0, \infty)$, (in fact $y \in$ $C^{1}[0, \infty)$ ), where $0 \leq y(t) \leq 1$ for all $t \in[0, \infty)$. Solving for $y$ we get

$$
y(t)=a(t) e^{-\int_{0}^{t} a(s) b(s) d s} \int_{0}^{t} b(s) e^{\int_{0}^{s} a(z) b(z) d z} d s,
$$

or equivalently,

$$
y(t)=\frac{\int_{0}^{t} b(s) e^{\int_{0}^{s} a(z) b(z) d z} d s}{\frac{1}{a(t)} e^{\int_{0}^{t} a(s) b(s) d s}} .
$$

We wish to examine $\lim _{t \rightarrow \infty} y(t)$. One particular case of interest is when $\lim _{t \rightarrow \infty} a(t)=0$ and $b(t)$ is nondecreasing. In this case (3.25) and L'Hôpital's Rule give

$$
\lim _{t \rightarrow \infty} y(t)=\lim _{t \rightarrow \infty} \frac{1}{1-\frac{a^{\prime}(t)}{a(t) b(t)}} .
$$

From (3.26) we can easily determine if $\lim _{t \rightarrow \infty} y(t)$ exists, and if so, what its value is. The family of kernels $a(t) b(s)=e^{-\alpha(t)} e^{\beta(s)}$ where $\alpha \in C^{1}[0, \infty)$ with $\alpha(t) \rightarrow \infty$ and $\beta \in C[0, \infty)$ with $\beta^{\prime}(t) \geq$ 0 for all $t \in[0, \infty)$, satisfy the above conditions and give

$$
\lim _{t \rightarrow \infty} y(t)=\lim _{t \rightarrow \infty} \frac{1}{1+\alpha^{\prime}(t) e^{-\beta(t)}} .
$$

[For applications of Theorem 3.1 and Theorem 3.2, recall that positive, separable kernels of the above type satisfy (3.7).]

Example 3.2. Consider

$$
y(t)=-A \int_{0}^{t} \frac{y(s)-\left(1-B e^{-s}\right)}{\sqrt{t-s}} d s
$$

where $A>0$ and $0 \leq B \leq 1$. Here $h \equiv 0, r(t)=1-B e^{-t}$ and $g(t, y)=y-1+B e^{-t}$. Now the conditions of Theorem 2.3 
are satisfied, therefore (3.27) has a solution $y \in C[0, \infty)$ with $0 \leq$ $y(t) \leq 1-B e^{-t}$. Solving for $y$ (see [8] for details) we get

where

$$
y(t)=\frac{e^{-A^{2} \pi t} g(t)+A^{2} \pi \int_{0}^{t} e^{-A^{2} \pi s} g(s) d s}{e^{-A^{2} \pi t}},
$$

$$
g(t):=A \int_{0}^{t} \frac{1-B e^{-s}}{\sqrt{t-s}} d s-A^{2} \pi \int_{0}^{t}\left(1-B e^{-s}\right) d s .
$$

Using l'Hôpital's Rule, one can see that $\lim _{t \rightarrow \infty} y(t)=1$.

Example 3.3. Consider

$$
y(t)=1-A \int_{0}^{t} \frac{y(s)-B e^{-s}}{\sqrt{t-s}} d s,
$$

where $A>0$ and $0 \leq B \leq 1$. Here $h \equiv 1, r(t)=B e^{-t}$ and $g(t, y)=y-B e^{-t}$. Therefore (3.28) satisfies the conditions of Theorem 2.2 and has a solution $B e^{-t} \leq y(t) \leq 1$, where

$$
y(t)=\frac{e^{-A^{2} \pi t} g(t)+A^{2} \pi \int_{0}^{t} e^{-A^{2} \pi s} g(s) d s}{e^{-A^{2} \pi t}},
$$

with

$$
g(t):=1-A^{2} B \pi-2 A \sqrt{t}+A^{2} B \pi e^{-t}+A B e^{-t} \int_{0}^{t} \frac{e^{s}}{\sqrt{s}} d s .
$$

It can be shown that $\lim _{t \rightarrow \infty} y(t)=0$. We omit the detail.

Equipped with these examples, we now give some applications of Theorem 3.1 and Theorem 3.2.

Example 3.4. Consider

$$
y(t)=1-\int_{0}^{t} \frac{y^{\frac{1}{n}}(s)}{\sqrt{t-s}} d s, t \in[0, \infty), n \in \mathbf{N}^{+} .
$$

Here $h=1, r=0$ and

$$
g(t, y)= \begin{cases}y^{\frac{1}{n}}, & y \geq 0 \\ 0, & y<0\end{cases}
$$


satisfy the hypotheses of Theorem 2.2, therefore (3.29) has a solution $y \in C[0, \infty)$ with $0 \leq y(t) \leq 1$.

If we can find a $\tilde{g}$ that satisfies (3.8) when $h \equiv 1$ and $k(t, s)=$ $\frac{1}{\sqrt{t-s}}$, then the hypotheses of Theorem 3.1 are satisfied, and it follows that $\lim _{t \rightarrow \infty} y(t)$ exists and $y(\infty)=0$.

Consider $\tilde{g}(y)=y$. Here $\tilde{r}=0$. Trivially $\tilde{g}(y)=y \leq y^{\frac{1}{n}}=$ $g(y)$ for $0 \leq y \leq 1$ and each $n \in \mathbf{N}^{+}$. Note also that

$$
y(t)=1-\int_{0}^{t} \frac{y(s)}{\sqrt{t-s}} d s
$$

is in fact (3.28) with $A=1$ and $B=0$. Therefore from Example 3.3 , (3.30) has a solution $\tilde{y} \in C_{l}[0, \infty)$, with $0 \leq \tilde{y}(t) \leq 1$ and $\tilde{y}(\infty)=0$. (3.8) is therefore satisfied and $y(\infty)=0$.

Example 3.5. Consider

$$
y(t)=-\int_{0}^{t} \frac{y^{\frac{1}{n}}(s)-1}{\sqrt{t-s}} d s, t \in[0, \infty), n \in \mathbf{N}^{+} .
$$

Since $h=0, r=1$ and

$$
g(t, y)= \begin{cases}y^{\frac{1}{n}}-1, & y \geq 0 \\ -1, & y<0\end{cases}
$$

we have from Theorem 2.3 that $(3.31)$ has a solution $y \in C[0, \infty)$ with $0 \leq y(t) \leq 1$.

We want to find a $\tilde{g}$ that satisfies (3.11). Consider $\tilde{g}(y)=\frac{1}{n}(y-1)$.

Here $\tilde{r}=1$. Since for $0 \leq y \leq 1$,

$$
y-1=\left(y^{\frac{1}{n}}-1\right)\left(1+y^{\frac{1}{n}}+y^{\frac{2}{n}}+\ldots+y^{\frac{n-1}{n}}\right) \geq n\left(y^{\frac{1}{n}}-1\right),
$$

we have that

$\tilde{g}(y)=\frac{1}{n}(y-1) \geq\left(y^{\frac{1}{n}}-1\right)=g(y)$ for each $n \in \mathbf{N}^{+}, 0 \leq y \leq 1$. 
Now

$$
y(t)=-\frac{1}{n} \int_{0}^{t} \frac{y(s)-1}{\sqrt{t-s}} d s, t \in[0, \infty)
$$

is (3.27) with $A=\frac{1}{n}$ and $B=0$. Therefore from Example 3.2, we have that (3.32) has a solution $\tilde{y} \in C[0, \infty)$ with $0 \leq \tilde{y}(t) \leq 1$ and $\lim _{t \rightarrow \infty} \tilde{y}(t)=1$. The hypotheses of Theorem 3.2 are satisfied when $\tilde{g}(y)=\frac{1}{n}(y-1)$, therefore $y(\infty)=1$.

Example 3.6. Consider

$$
y(t)=-A \int_{0}^{t} \frac{y^{n}(s)-\left(1-B e^{-s}\right)}{\sqrt{t-s}} d s, t \in[0, \infty)
$$

with $A>0,0 \leq B \leq 1$ and $n \geq 1$. Here $h \equiv 0$,

$$
g(t, y)= \begin{cases}-A\left(y^{n}-\left(1-B e^{-t}\right)\right), & y \geq 0 \\ A\left(1-B e^{-t}\right), & y<0\end{cases}
$$

and $r(t)=\left(1-e^{-t}\right)^{\frac{1}{n}}$. For a comparison equation consider (3.27). This implies that $\tilde{g}(t, y)=-A\left(y-\left(1-B e^{-t}\right)\right)$ and $\tilde{r}(t)=1-e^{-t}$. It is easy to check that Theorem 3.2 is satisfied with this choice of $\tilde{g}$, therefore (3.33) has a solution $y \in C[0, \infty)$, with $0 \leq y(t) \leq$ $\left(1-e^{-t}\right)^{\frac{1}{n}}$ and $y(\infty)=1$.

Example 3.7. Finally consider

$$
y(t)=-\int_{0}^{t} A(s) \frac{\left[y^{n}(s)-\left(1-B e^{-s}\right)\right]}{\sqrt{t-s}} d s
$$

where $A \in C[0, \infty)$ with $A(t) \geq A>0$ for all $t \in[0, \infty), 0 \leq$ $B \leq 1$. Compare (3.34) with (3.33). By Theorem 3.2, (3.34) has a solution $y \in C[0, \infty)$ with $0 \leq y(t) \leq\left(1-e^{-t}\right)^{\frac{1}{n}}$ and $y(\infty)=1$.

Remark 3.3. We could have used Theorem 3.3 in Example 3.2 and Example 3.6, but note that Theorem 3.3 does not apply to Examples 3.1, 3.3 - 3.5 and 3.7. 


\section{References}

[1] C. Corduneanu, Integral Equations and Stability of Feedback Systems. Academic Press: New York, 1973.

[2] C. Corduneanu, Integral Equations and Applications. Cambridge Univ. Press: New York, 1990

[3] A. Friedman, On integral equations of Volterra type, J. Anal. Math. 11 (1963), 381-413.

[4] G. Gripenberg, S. O. Londen and O. Staffans, Volterra Integral and Functional Equations. Cambridge Univ. Press: New York, 1990.

[5] H. Hochstadt, Integral Equations. John Wiley: New York, 1973.

[6] M. A. Krasnoselskii, Topological Methods in the Theory of Nonlinear Integral Equations. Pergamon Press: Oxford, 1964.

[7] J. W. Lee and D. O'Regan, Existence principles for nonlinear integral equations on semi-infinite and half-open intervals, in Advances in Nonlinear Dynamics, ed. by S. Sivasubdarem and A. A. Martynyuk, 355-364. Gordon \& Breach Science Publishers Ltd.: Amsterdam, 1997.

[8] M. Meehan and D. O'Regan, Existence theory for nonlinear Fredholm and Volterra integral equations on half open intervals, Nonlinear Analysis 35 (1999), 355-387.

[9] R. K. Miller, On Volterra integral equations with nonnegative integrable resolvents, J. Math. Anal. Appl. 22 (1968), 319-340.

[10] D. O'Regan, Existence results for nonlinear integral equations, J. Math. Anal. Appl. 192 (1995), 705-726.

[11] D. O'Regan, Integral inclusions of upper semi-continuous and lower semi-continuous type, Proc. A.M.S. 124 (1996), 2391-2399.

[12] R. L. Wheeden and A. Zygmund, Measure and Integral. Marcel Dekker, Inc.: New York and Basel, 1977.

[13] K. Yosida, Functional Analysis (5th ed). Springer-Verlag: Berlin, 1978.

Maria Meehan

Department of Mathematics

National University of Ireland, Dublin

Belfield

Dublin 4

Donal O'Regan

National University of Ireland, Galway

Galway 